\title{
Web-Based Health Information Technology: Access Among Latinos Varies by Subgroup Affiliation
}

\author{
Mariaelena Gonzalez ${ }^{1,2^{*}}, \mathrm{PhD}$; Ashley Sanders-Jackson ${ }^{3 *}, \mathrm{PhD}$; Tashelle Wright ${ }^{1}, \mathrm{MSPH}$ \\ ${ }^{1}$ Department of Public Health, University of California - Merced, Merced, CA, United States \\ ${ }^{2}$ Health Science Research Institute, University of California - Merced, Merced, CA, United States \\ ${ }^{3}$ Department of Advertising and Public Relations, College of Communication Arts and Sciences, Michigan State University, East Lansing, MI, United \\ States \\ these authors contributed equally
}

\section{Corresponding Author:}

Mariaelena Gonzalez, PhD

Department of Public Health

University of California - Merced

5200 N Lake Road

Merced, CA,

United States

Phone: 16502838307

Email: mgonzalez82@ucmerced.edu

\section{Abstract}

Background: There are significant health technology gaps between Latinos and non-Hispanic whites and between first- and second-generation Latinos.

Objective: This study aimed to examine disparities in Web-based health information-seeking behavior (HISB) and patient portal use among Latinos, taking into account nativity and subethnic affiliation.

Methods: We analyzed US-born, non-Hispanic whites and Latinos adults $(\mathrm{N}=49,259)$ and adult internet users $(\mathrm{N}=36,214)$ in the 2015 to 2016 National Health Interview Survey using a binary logistic regression controlling for individual difference level variables. Outcomes were internet use, HISB (health information-seeking online and using a chat group for health information), and patient portal use (using a computer to schedule an appointment, filling a prescription, and communicating with a provider).

Results: We found that US-born Mexicans (odds ratio [OR] 0.81, 95\% CI 0.66-0.99), foreign-born Mexicans (OR 0.35, 95\% CI 0.29-0.42), foreign-born Puerto Ricans (OR 0.62, 95\% CI 0.44-0.87), foreign-born Central and South Americans (OR 0.42, 95\% CI 0.33-0.53), and foreign-born other Latinos (OR 0.34, 95\% CI 0.24-0.49) had lower odds of using the internet than US-born non-Hispanic whites. The relationship between subgroup affiliation and Web-based HISB varied by type of technology. US-born Mexicans (OR 0.77, 95\% CI 0.66-0.9), foreign-born Mexicans (OR 0.51, 95\% CI 0.43-0.61), foreign-born Central and South Americans (OR 0.53, 95\% CI 0.43-0.64), and foreign-born other Latinos (OR 0.56, 95\% CI 0.4-0.79) had lower odds of looking up health information online than US-born non-Hispanic whites. Controlling for age, sex, education, income to federal poverty level, and region, foreign-born Central and South Americans (OR 0.61, 95\% CI 0.41-0.92) and foreign-born other Latinos (OR $0.26,95 \%$ CI 0.1-0.68) had lower odds of filling a prescription using a computer than US-born non-Hispanic whites. Foreign-born Mexicans (OR 0.51, 95\% CI 0.36-0.72) and foreign-born Central and South Americans (OR 0.7, 95\% CI 0.5-0.99) have lower odds of emailing a health care provider than US-born non-Hispanic whites. Posthoc analyses were conducted among Mexican-Americans to see if age was significant in predicting Web-based HISB or other patient portal use. We found individuals aged 18 to 30 years had higher odds of using the internet (OR 3.46, 95\% CI 2.61-4.59) and lower odds of looking up health information online (OR $0.75,95 \%$ CI $0.58-0.96$ ). A posthoc analysis was conducted among Mexican-Americans to see if nativity predicted Web-based HISB and patient portal use. We found that US-born individuals had higher odds (OR 52.9, 95\% CI 1.2-1.93) of looking up health information online compared with foreign-born individuals.

Conclusions: We found Latino subgroups do not use health information channels equally, and attempts to target Latinos should take ethnicity and nativity into account.

(J Med Internet Res 2019;21(4):e10389) doi: $\underline{10.2196 / 10389}$ 


\section{KEYWORDS}

information seeking behavior; health information technology; Hispanic Americans; minority health

\section{Introduction}

\section{Background}

The use of Web-based health information technology (HIT) is spreading [1]. For example, encouraged by the Health Information Technology for Economic and Clinical Health Act, in 2012, at least half of all health care providers had adopted the use of patient portals [2]. Patient portals have been shown to have a wide variety of benefits, including the management of chronic disease; however, Latinos are also less likely to adopt patient portals $[3,4]$. Previous research has suggested disparities in internet access, online health information-seeking behavior (HISB) between non-Hispanic (NH) whites and Latinos, and between US- and foreign-born Latinos [5-11]. With the increasing reliance on patient portals [4], it is important to understand where disparities in specific types of online HISB and patient portal use occur for Latinos at the national level.

Although Latinos have historically lagged behind $\mathrm{NH}$ whites in access to the internet, there have been significant gains in internet access among Latinos between 2012 and 2015, particularly among Spanish speakers [8]. Despite this, only 46\% of Hispanics access the internet through broadband, and a 2017 Pew Research Report stated that $22 \%$ of Hispanics did not have broadband but owned a smartphone $[8,12]$. These differential patterns of access and device use are disparities, and disparities in the use of Web-based HIT, such as patient portals, should also be investigated.

There are significant information knowledge gaps between Latinos and NH whites when it comes to Web-based patient portals [4,13-15]. In a systematic review of patient portal literature, although patient portals may improve outcomes, particularly in conjunction with a clinical intervention, for some patients with chronic conditions, racial and ethnic minorities may not receive the same benefit as whites [15]. Another systematic review found that ethnicity can play a role in lack of adoption of patient portals [4]. Indeed, in a California sample of elderly adults, Latinos were less likely to use patient portals than NH whites, although Latinos were not broken down into subgroups in this analysis [16]. Latinos also have lower rates of activating and logging into patient portals than $\mathrm{NH}$ whites $[17,18]$.

Although some state- and local-level examinations of online HISB and the use of patient portals exist [9,19-22], and several national level studies [1,11], these studies do not control for other factors such as Latino subethnicity or nativity that might be important cultural variables that could contribute to or mitigate disparities.

When examining Latinos in the United States, nativity and ethnicity are important determinants of health and media use. Although Latinos are the largest ethnic group in the United States [23], there are significant demographic differences between US- and foreign-born individuals. On average, US-born Latinos, who make up over $60 \%$ of the Latino population in the
United States, are younger and consume more English-language media [8,24]. US-born Latinos engage in more online HISB and have higher levels of confidence in their ability to fill out Web-based forms than foreign-born Latinos [9]. Although researchers commonly lump Latino subethnicities together, there are significant health and behavioral differences within and between the various subethnicities [25-28]. For example, research suggests that Puerto Ricans are more likely to be current smokers and to have ever smoked compared with Dominicans, Colombians, and Ecuadorian individuals [27]. Puerto Ricans also reportedly have higher rates of depression and suicide attempts compared with Mexican and Cuban Americans [26], Cuban Americans have higher systolic blood pressure and higher cholesterol than both Mexican Americans and Puerto Ricans [25], and perceptions of cancer risk factors vary across Latino ethnic subgroups [28]. A study in 2007, which examined internet use among Latinos by subethnicity, found that in the United States, internet use varies among ethnic groups, with South American origin Latinos having a higher than average rate of internet use and Mexicans and Central Americans having lower rates of internet use [29], which indicates that ethnicity is an important factor when examining internet-related behavior among Latinos. However, we did not find other studies on Latino internet use that disaggregate Latinos in this way. Due to the US or foreign-born (nativity) and ethnic differences among Latinos, it is important to take into account nativity and ethnicity, if possible, when examining disparities between $\mathrm{NH}$ whites and Latinos.

\section{Objective}

The purpose of this study was to examine disparities in online HISB and patient portal use among NH whites, US-born Latinos, and foreign-born Latinos, taking into account subethnic affiliation and disaggregating subethnicities or regional groupings when possible. By understanding where disparities exist, we can effectively target interventions to increase online health literacy within the largest linguistically and culturally sensitive ethnic group in the United States.

\section{Methods}

\section{Data}

We combined 2 years (2015 and 2016) of the adult sample file of the National Health Interview Survey (NHIS), which is a nationally representative sample of the adult US civilian population. We examined all US-born whites and Latinos $(\mathrm{N}=49,251)$ and only those individuals who reported using the internet $(\mathrm{N}=36,214)$.

\section{Primary Outcomes}

Respondents were asked if in the last 12 months they had used the internet or used email. In addition, they were asked if in the last year they had engaged in online HISB and used a computer to (1) "[1]ook up health information on the Internet," [30] or (2) "[u]se online chat groups to learn about health topics" [30]. They were also asked if they had used patient portals by using 
a computer to (3) "[f]ill a prescription," [30] (4) "[s]chedule an appointment with a health care provider," [30] or (5) "[c]ommunicate with a health care provider by email" [30]. Each of these binary items was separately used as a primary outcome in our analyses. If respondents reported using the internet, email, engaging in online HISB or online patient portals in the last 12 months, they were classified as having used the internet in the last 12 months.

\section{Latino Subgroup Affiliation by Nativity}

There are differences between US- and foreign-born Hispanics with regard to the median age and use of Spanish or English within the population $[9,24,31,32]$. There are differences between Hispanic subgroups and region, which may play a role in health care access [25-28]. Furthermore, different countries of origin may be associated with cultural differences that impact health access. In addition, studies have shown significant behavioral and health differences among various Hispanic ethnic groups, including around internet use [29,31-33]. Although combining 2 years of the NHIS did not yield a large enough sample to disaggregate all Latino ethnic subgroups in our model, we were able to obtain a large enough sample to divide Hispanics into the following subgroups: Mexican and Mexican-Americans (Mexicans), Puerto Ricans, Central and South American, and other Latino (Cuban, Dominican, Other Latin American affiliation). Although some subgroups we used contain large regions or disparate groups, we combined these groups based on Centers for Disease Control and Prevention's classifications and because of sample size issues. Individuals who identified as other Spanish or multiple Hispanic were excluded as we could not specifically identify their ethnic or regional origin. We further divided each of the 4 Latino subgroups into US- and foreign-born individuals. Our comparison group for all Latino categories was US-born $\mathrm{NH}$ whites, as it is possible that foreign-born $\mathrm{NH}$ whites have different health outcomes and media use patterns than US-born individuals.

\section{Other Individual-Level Variables}

We controlled for age (18-30 years, 31-54 years, and 55 years and above), gender (male, female), education (below a high school education, completed high school or general education development, some college, and completed a bachelor's or higher), poverty level $(0 \%-99 \%$ of the federal poverty level [FPL], $100 \%-199 \%$ of the FPL, and $200 \%$ or greater of the FPL), marital status (married or partnered, unpartnered), and whether or not the respondent was insured. We also controlled for the individuals' occupational group, classifying individuals who were employed by their 4-category occupational group status (white collar, blue collar, farm and forestry, and service) and those who were not in the labor force (individuals who reported that they were looking for work, or not working at a job or business, and not looking for work). We could not use this 5-category occupational and employment designations in our posthoc statistical analyses as 138 of the 170 Mexican-descent individuals in the farm, or fishing or forestry category were foreign-born, which means there was little variation in this category among Mexican-identified individuals, so we combined this category with the blue-collar category in the posthoc analyses. In addition, we controlled for region of the United States (North, Midwest, South, and West) and whether or not the respondent reported using the internet at least once a day. To minimize collinearity, we did not control for English proficiency, as we found it significantly negatively correlated with being US-born among our sample of US-born whites and Latinos (correlation=-0.66).

\section{Statistical Analysis}

We ran multivariable binary logistic regressions to test the relationship between ethnicity and nativity and internet use. We then used a binary logistic regression to test the relationship between ethnicity and nativity and our 2 HISB and 3 patient portal outcomes among internet users. The sample was weighted according to survey directions. Due to the small number of individuals among some of the Latino ethnic and nativity groups, we do not report groups where too few individuals use the technology in question to draw a statistical conclusion.

\section{Results}

\section{Demographics}

The majority $(38,256 / 49,259,82.6 \%$; 95\% CI 82.1-83.2) of respondents reported using the internet (Table 1), and of $65.05 \%$ (25,209/38,256; 95\% CI 64.3-65.8) respondents reported looking up health information on the internet; however, less than $13 \%$ of respondents reported using a computer to engage in any of the surveyed Web-based patient portal channels (Table 2). Among internet users, Mexican-identified individuals (Mexicans) were the largest US- and foreign-born subgroups (2337/38,256, 6.24\%; 95\% CI 5.8-6.8 and 1358/38,256, 4.1\%; 95\% CI 3.8-4.4, respectively). The next largest US-born subgroup was Puerto Ricans (414/38,256, 1.2\%; 95\% CI $0.97-1.24)$. There were more foreign-born Central and South Americans $(739 / 38,256)$ and other Latinos $(307 / 38,256)$ than US-born individuals within these subgroupings $(246 / 38,256$ and 139/38,256, respectively). 
Table 1. Characteristics of US-born non-Hispanic whites and Latinos (National Health Interview Survey, 2015-16). N=49,259.

\begin{tabular}{|c|c|c|}
\hline Variables & Statistics, n (\%) & $95 \% \mathrm{CI}$ \\
\hline \multicolumn{3}{|l|}{ Dependent variables } \\
\hline Use the internet & $38,256(82.61)$ & $82.05-83.15$ \\
\hline \multicolumn{3}{|l|}{ Independent variables } \\
\hline \multicolumn{3}{|c|}{ Ethnicity by nativity $\mathrm{NH}^{\mathrm{a}}$ whites and Latino subgroup affiliation } \\
\hline US-born NH White & $40,732(80.36)$ & $79.34-81.34$ \\
\hline US-born Mexican & $2585(6.06)$ & $5.56-6.79$ \\
\hline Foreign-born Mexican & $2718(6.28)$ & $5.8-6.79$ \\
\hline US-born Puerto Rican & $489(1.09)$ & $0.97-1.24$ \\
\hline Foreign-born Puerto Rican & $476(0.99)$ & $086-1.14$ \\
\hline US-born Central or South American & $268(0.71)$ & $0.59-0.85$ \\
\hline Foreign-born Central or South American & $1195(2.86)$ & $2.59-3.16$ \\
\hline US-born other Latino & $157(0.42)$ & $0.33-0.52$ \\
\hline Foreign-born other Latino & $631(1.22)$ & $1.04-1.43$ \\
\hline \multicolumn{3}{|l|}{ Age, years } \\
\hline $18-30$ & $8837(22.21)$ & $21.58-22.85$ \\
\hline $31-54$ & $18,386(40.29)$ & $39.65-40.94$ \\
\hline$>55$ & $22,036(37.5)$ & $36.81-38.19$ \\
\hline \multicolumn{3}{|l|}{ Sex } \\
\hline Female & $26,633(51.18)$ & $50.57-51.79$ \\
\hline Male & $22,626(48.82)$ & $48.21-49.43$ \\
\hline \multicolumn{3}{|l|}{ Education } \\
\hline Below high school & $6152(12.37)$ & $11.87-12.88$ \\
\hline High school or general education development & $12,258(24.92)$ & $24.33-25.52$ \\
\hline Some college & $15,633(31.53)$ & $30.91-32.16$ \\
\hline Bachelor's or above (reference) & $15,072(31.18)$ & $30.41-31.96$ \\
\hline \multicolumn{3}{|l|}{ Income to federal poverty level (FPL) ratio } \\
\hline Poor (income to FPL ratio 0-0.99) & $6266(10.66)$ & $10.22-11.12$ \\
\hline Near poor (income to FPL ratio 1-1.99) & $8843(17.32)$ & $16.8-17.85$ \\
\hline Above poor (income to FPL ratio >2) & $31,788(72.02)$ & $71.31-72.73$ \\
\hline \multicolumn{3}{|l|}{ Married or partnered } \\
\hline Married or partnered & $26,135(63.48)$ & $62.83-64.13$ \\
\hline Single or widowed & $23,040(36.52)$ & $35.87-37.17$ \\
\hline \multicolumn{3}{|l|}{ Occupational group } \\
\hline White collar & $28,234(58.12)$ & $57.39-58.85$ \\
\hline Service & $7615(15.37)$ & $14.89-15.86$ \\
\hline Farm and forestry & $475(0.86)$ & $0.73-1.01$ \\
\hline Blue Collar & $10,168(21.03)$ & $20.46-21.61$ \\
\hline Not in labor force & $2138(4.63)$ & $4.32-4.96$ \\
\hline \multicolumn{3}{|l|}{ Health insurance } \\
\hline Covered & $44,471(90.16)$ & $89.71-90.6$ \\
\hline Not covered & $4613(9.84)$ & $9.4-10.29$ \\
\hline
\end{tabular}




\begin{tabular}{lll}
\hline Variables & Statistics, n (\%) & $95 \%$ CI \\
\hline Region & & $16.67-18.35$ \\
Northeast & $8473(17.49)$ & $23.66-25.38$ \\
Midwest & $11,801(24.51)$ & $33.27-35.64$ \\
South & $15,617(34.44)$ & $22.45-24.69$ \\
West & $13,368(23.55)$ & \\
\hline
\end{tabular}

${ }^{\mathrm{a}} \mathrm{NH}$ : non-Hispanic.

\section{Internet Use}

The percentage of internet users who use each technology varies by ethnicity and nativity (Tables 3-5). A total of $85.6 \%$ $(32,735 / 40,732 ; 95 \%$ CI 85.1-86.1) of US-born NH whites report using the internet, whereas $53.76 \%$ (1358/2037; 95\% CI 51.1-56.4) of US-born Mexicans reported using the internet. Foreign-born Mexicans (633/2037) and foreign-born Central and South Americans (370/739) and foreign-born other Latinos $(145 / 307)$ had less than $50 \%$ of the population report looking up health information online.

We found that US-born Mexicans (OR 0.81, 95\% CI 0.66-0.99), foreign-born Mexicans (OR 0.35, 95\% CI 0.29-0.42), foreign-born Puerto Ricans (OR 0.62, 95\% CI 0.44-0.87), foreign-born Central and South Americans (OR 0.42, 95\% CI 0.33-0.53), and foreign-born other Latinos (OR 0.34, 95\% CI 0.24-0.49) had lower odds of using the internet than US-born NH whites (Table 6).

\section{Online Health Information-Seeking Behavior}

The relationship between subgroup affiliation and online HISB varied by type of technology. US-born Mexicans (OR 0.77,
95\% CI 0.66-0.90), foreign-born Mexicans (OR 0.51, 95\% CI 0.43-0.61), foreign-born Central and South Americans (OR $0.53,95 \%$ CI $0.43-0.64$ ), and foreign-born other Latinos (OR $0.56,95 \%$ CI $0.40-0.79$ ) had lower odds of looking up health information online than US-born $\mathrm{NH}$ whites (Table 6). Foreign-born Central and South Americans (OR 1.71, 95\% CI 1.11-2.62) had higher odds of using a chat group to discuss health information than US-born $\mathrm{NH}$ whites, whereas there was no significant difference between all other groups and $\mathrm{NH}$ whites regarding the use of chat groups to discuss health information.

\section{Patient Portal Use}

Controlling for age, sex, education, income to FPL, and region, foreign-born Central and South Americans (OR 0.61, 95\% CI 0.41-0.92) had lower odds of filling a prescription using a computer than US-born NH whites (Table 7). There was no significant difference using a computer to schedule medical appointments between US-born NH whites and the various Latino ethnic and nativity groups. Foreign-born Mexicans (OR $0.51,95 \%$ CI 0.36-0.72) and foreign-born Central and South Americans (OR 0.51, 95\% CI 0.36-0.72) have lower odds of emailing a health care provider than US-born $\mathrm{NH}$ whites. 
Table 2. Characteristics of US-born non-Hispanic whites and Latino internet users (National Health Interview Survey, 2015-16). N=38,256.

\begin{tabular}{|c|c|c|}
\hline Variables & Statistics, n (\%) & $95 \% \mathrm{CI}$ \\
\hline \multicolumn{3}{|l|}{ Dependent variables } \\
\hline Use the internet & $-^{\mathrm{a}}$ & - \\
\hline \multicolumn{3}{|l|}{ Online health information-seeking behavior } \\
\hline Look up health information on the internet & $25,209(65.05)$ & $64.3-65.8$ \\
\hline Use online chat groups to learn about health topics & $1634(4.32)$ & $4.06-4.59$ \\
\hline \multicolumn{3}{|l|}{ Web-based health information technology } \\
\hline Used a computer to fill a prescription & $4564(11.91)$ & $11.43-12.41$ \\
\hline Used a computer to schedule an appointment with a health care provider & $4834(13.43)$ & $12.87-14.01$ \\
\hline Used a computer to communicate with a health care provider by email & $5653(15.2)$ & $14.6-15.82$ \\
\hline \multicolumn{3}{|l|}{ Independent variables } \\
\hline \multicolumn{3}{|l|}{ Ethnicity by nativity $\mathrm{NH}^{\mathrm{b}}$ whites and Latino subgroup affiliation } \\
\hline US-born NH white & $32,735(83.28)$ & $82.41-84.11$ \\
\hline US-born Mexican & $2337(6.24)$ & $5.75-6.76$ \\
\hline Foreign-born Mexican & $1358(4.08)$ & $3.76-4.44$ \\
\hline US-born Puerto Rican & $414(1.18)$ & $1.03-1.35$ \\
\hline Foreign-born Puerto Rican & $276(0.78)$ & $0.66-0.93$ \\
\hline US-born Central and South American & $246(0.82)$ & $0.68-0.99$ \\
\hline Foreign-born Central and South American & $739(2.33)$ & $2.07-2.63$ \\
\hline US-born other Latino & $139(0.46)$ & $0.37-0.59$ \\
\hline Foreign-born other Latino & $307(0.8)$ & $0.67-0.96$ \\
\hline \multicolumn{3}{|l|}{ Age, years } \\
\hline $18-30$ & $8191(25.37)$ & $24.64-26.12$ \\
\hline $31-54$ & $15,772(42.97)$ & $42.26-43.69$ \\
\hline$>55$ & $14,293(31.66)$ & 30.94-32.39 \\
\hline \multicolumn{3}{|l|}{ Sex } \\
\hline Female & $20,790(51.51)$ & $50.83-52.19$ \\
\hline Male & $17,466(48.49)$ & 47.81-49.19 \\
\hline \multicolumn{3}{|l|}{ Education } \\
\hline Below high-school & $2488(7.09)$ & $6.71-70.5$ \\
\hline High-school or general education development & $8253(22.20)$ & $21.59-22.83$ \\
\hline Some college & $13,348(34.26)$ & $33.54-34.98$ \\
\hline Bachelor's or above (reference) & $14,106(36.45)$ & $35.58-37.32$ \\
\hline \multicolumn{3}{|l|}{ Income to federal poverty level (FPL) ratio } \\
\hline Poor (income to FPL ratio $0-0.99$ ) & $3899(8.41)$ & 7.99-8.86 \\
\hline Near poor (income to FPL ratio 1-1.99) & $5630(14.34)$ & $13.81-14.88$ \\
\hline Above poor (income to FPL ratio >2) & $27,237(77.25)$ & $76.55-77.93$ \\
\hline \multicolumn{3}{|l|}{ Married or partnered } \\
\hline Married or partnered & $21,536(65.36)$ & $64.63-66.09$ \\
\hline Single or widowed & $16,664(34.64)$ & 33.91-35.37 \\
\hline \multicolumn{3}{|l|}{ Occupational group } \\
\hline White collar & $24,659(64.45)$ & $63.71-65.18$ \\
\hline
\end{tabular}




\begin{tabular}{lll}
\hline Variables & Statistics, $\mathrm{n}(\%)$ & $95 \%$ CI \\
\hline Service & $5403(14.28)$ & $13.76-14.81$ \\
Farm and forestry & $228(0.51)$ & $0.42-0.6$ \\
Blue collar & $6452(17.48)$ & $3.01-3.59$ \\
$\quad$ Not in labor force & $1028(3.29)$ & $77.41-79.21$ \\
Use the internet once a day or more often & & $20.79-22.59$ \\
Use the internet once a day or more often & $28,591(78.32)$ & $90.78-91.62$ \\
Use the internet less than once a day & $7804(21.68)$ & $8.38-9.22$ \\
Health insurance & $34,801(91.21)$ & $16.8-18.59$ \\
Covered & $3319(8.79)$ & $23.97-25.86$ \\
Not covered & & $32.07-34.5$ \\
Region & $6576(17.68)$ & $23.02-25.31$ \\
Northeast & $9273(24.9)$ & $11,653(33.27)$ \\
Midwest & $10,772(24.15)$ & \\
South & & \\
West & & \\
\hline
\end{tabular}

a_: not applicable.

${ }^{\mathrm{b}} \mathrm{NH}$ : non-Hispanic.

Table 3. Percent of individuals in each ethnic-native group who use the internet and look up health information online (National Health Interview Survey, 2015-16).

\begin{tabular}{|c|c|c|c|c|}
\hline \multirow[t]{2}{*}{ Ethnicity by nativity } & \multicolumn{2}{|c|}{ Population: use the internet } & \multicolumn{2}{|c|}{ Population: look up health information } \\
\hline & $\mathrm{n}(\%)$ & $95 \% \mathrm{CI}$ & $\mathrm{n}(\%)$ & $95 \% \mathrm{CI}$ \\
\hline US-born non-Hispanic whites & $32,735(85.6)$ & $85.1-86.1$ & $22,167(67.3)$ & $66.6-68.1$ \\
\hline US-born Mexican & $2037(85.0)$ & $82.7-87.1$ & $1228(58.2)$ & $55.0-61.4$ \\
\hline Foreign-born Mexican & $1358(53.8)$ & $51.1-56.4$ & $633(45.1)$ & $41.6-48.7$ \\
\hline US-born Puerto Rican & $414(88.9)$ & $84.0-92.4$ & $247(58.2)$ & $52.1-64.0$ \\
\hline Foreign-born Puerto Rican & $276(65.2)$ & $60.2-70.0$ & $155(56.3)$ & $48.2-64.0$ \\
\hline US-born Central and South American & $246(95.9)$ & $92.5-97.7$ & $171(69.1)$ & $60.6-76.5$ \\
\hline Foreign-born Central and South American & $739(67.3)$ & $63.6-70.8$ & $370(48.8)$ & $44.1-53.5$ \\
\hline US-born other Latino & $139(91.4)$ & $83.7-95.6$ & $90(61.7)$ & $51.1-71.3$ \\
\hline Foreign-born other Latino & $307(55.4)$ & $50.0-60.7$ & $145(45.8)$ & $38.8-52.9$ \\
\hline
\end{tabular}


Table 4. Percent of individuals in each ethnic-native group who are internet users and use a chat group or fill a prescription online (National Health Interview Survey, 2015-16).

\begin{tabular}{|c|c|c|c|c|}
\hline \multirow[t]{2}{*}{ Ethnicity by nativity } & \multicolumn{2}{|c|}{ Population: use chat group } & \multicolumn{2}{|c|}{ Population: fill prescription } \\
\hline & $\mathrm{n}(\%)$ & $95 \% \mathrm{CI}$ & $\mathrm{n}(\%)$ & $95 \% \mathrm{CI}$ \\
\hline US-born whites & $1349(4.2)$ & $4.0-4.5$ & $4170(12.9)$ & $12.3-13.4$ \\
\hline US-born Mexican & $93(4.1)$ & $3.1-5.4$ & $184(9.2)$ & $7.7-11.2$ \\
\hline Foreign-born Mexican & $73(4.7)$ & $3.6-6.2$ & $62(5.0)$ & $3.7-6.7$ \\
\hline US-born Puerto Rican & $21(3.4)$ & $2.0-5.8$ & $37(8.2)$ & $5.0-13.2$ \\
\hline Foreign-born Puerto Rican & $15(6.4)$ & $3.2-12.5$ & $19(6.0)$ & $3.5-9.9$ \\
\hline US-born Central and South American & $12(3.5)$ & $1.7-6.9$ & $30(8.9)$ & $5.5-14.0$ \\
\hline Foreign-born Central and South American & $52(8.0)$ & $5.6-11.3$ & $46(6.5)$ & $4.5-9.2$ \\
\hline US-born other Latino & $3(2.1)$ & $0.5-8.4$ & $8(4.3)$ & $1.9-9.4$ \\
\hline Foreign-born other Latino & $16(3.9)$ & $2.0-7.5$ & $7(2.3)$ & $1.0-5.3$ \\
\hline
\end{tabular}

Table 5. Percent of individuals in each ethnic-native group who use the internet and look up health information online (National Health Interview Survey 2015-16).

\begin{tabular}{|c|c|c|c|c|}
\hline \multirow[t]{2}{*}{ Ethnicity by nativity } & \multicolumn{2}{|c|}{ Population: schedule medical appointment } & \multicolumn{2}{|c|}{ Population: email providers } \\
\hline & $\mathrm{n}(\%)$ & $95 \% \mathrm{CI}$ & $\mathrm{n}(\%)$ & $95 \% \mathrm{CI}$ \\
\hline US-born whites & $4278(14.1)$ & $13.5-14.7$ & $5177(16.5)$ & $15.9-17.2$ \\
\hline US-born Mexican & $247(12.7)$ & $10.8-14.8$ & $209(10.9)$ & $9.1-13.1$ \\
\hline Foreign-born Mexican & $89(6.8)$ & $5.3-8.7$ & $66(4.8)$ & $3.5-6.5$ \\
\hline US-born Puerto Rican & $47(12.2)$ & $8.4-17.2$ & $46(9.3)$ & $6.3-13.5$ \\
\hline Foreign-born Puerto Rican & $25(7.9)$ & $5.0-12.3$ & $17(6.0)$ & $3.6-9.9$ \\
\hline US-born Central and South American & $47(17.1)$ & $11.7-24.3$ & $40(16.2)$ & $10.5-24.2$ \\
\hline Foreign-born Central and South American & $71(9.5)$ & $7.2-12.4$ & $71(9.4)$ & $7.0-12.5$ \\
\hline US-born other Latinos & $13(10.4)$ & $5.8-17.7$ & $11(5.9)$ & $3.0-11.3$ \\
\hline Foreign-born other Latinos & $17(5.3)$ & $3.1-8.9$ & $16(5.6)$ & $2.9-10.6$ \\
\hline
\end{tabular}


Table 6. Correlation between (1) Latino subgroup affiliation and internet use and (2) Latino subgroup affiliation and online health information seeking behavior among internet users, controlling for age, sex, education, income to federal poverty level, marital or partnered status, occupation, insurance status, daily internet use, and region using the National Health Interview Survey 2015-16.

\begin{tabular}{|c|c|c|c|}
\hline Variables $^{\mathrm{a}}$ & $\begin{array}{l}\text { Dependent variable: use internet } \\
(\mathrm{n}=45,449), \mathrm{OR}^{\mathrm{b}}(95 \% \mathrm{CI})\end{array}$ & $\begin{array}{l}\text { Internet users } \\
\text { Dependent variable: look up health } \\
\text { information }(\mathrm{n}=34,467), \mathrm{OR}(95 \% \\
\text { CI) }\end{array}$ & $\begin{array}{l}\text { Dependent variable: use chat group } \\
(\mathrm{n}=34,484), \text { OR }(95 \% \mathrm{CI})\end{array}$ \\
\hline US-born white & 1.00 (Reference) & 1.00 (Reference) & 1.00 (Reference) \\
\hline US-born Mexican & $0.81(0.66-0.99)^{\mathrm{c}}$ & $0.77(0.66-0.9)^{\mathrm{c}}$ & $1.02(0.74-1.42)$ \\
\hline US-born Puerto Rican & $1.2(0.78-1.84)$ & $0.75(0.56-1.01)$ & $0.96(0.54-1.7)$ \\
\hline $\begin{array}{l}\text { US-born Central and South Ameri- } \\
\text { can }\end{array}$ & $1.44(0.75-2.74)$ & $1.16(0.76-1.77)$ & $0.92(0.44-1.91)^{\mathrm{d}}$ \\
\hline US-born other Latino & $1(0.41-2.47)$ & $0.83(0.49-1.4)$ & $0.5(0.11-2.18)^{\mathrm{d}}$ \\
\hline Foreign-born Mexican & $0.35(0.29-0.42)^{\mathrm{c}}$ & $0.51(0.43-0.61)^{\mathrm{c}}$ & $1.28(0.9-1.82)$ \\
\hline Foreign-born Puerto Rican & $0.62(0.44-0.87)^{\mathrm{c}}$ & $0.82(0.57-1.17)$ & $1.27(0.54-2.99)$ \\
\hline $\begin{array}{l}\text { Foreign-born Central and South } \\
\text { American }\end{array}$ & $0.42(0.33-0.53)^{\mathrm{c}}$ & $0.53(0.43-0.64)^{\mathrm{c}}$ & $1.71(1.11-2.62)^{\mathrm{c}}$ \\
\hline Foreign-born other Latino & $0.34(0.24-0.49)^{\mathrm{c}}$ & $0.56(0.4-0.79)^{\mathrm{c}}$ & $0.92(0.43-1.97)^{\mathrm{d}}$ \\
\hline
\end{tabular}

${ }^{\mathrm{a} C}$ Control variables not listed include age, sex, education, income to federal poverty level ratio, occupational group, insurance status, daily internet use, and region.

${ }^{\mathrm{b}} \mathrm{OR}$ : odds ratio.

${ }^{\mathrm{c}}$ Indicates values are significant.

${ }^{\mathrm{d}}$ Too few individuals use the technology in question to draw a statistical conclusion.

Table 7. Correlation between Latino subgroup affiliation and online patient portal use among internet users controlling for age, sex, education, income to federal poverty level, marital or partnered status, occupation, insurance status, daily internet use, and region using the National Health Interview Survey 2015-16. N=46,480.

\begin{tabular}{|c|c|c|c|}
\hline \multirow[t]{2}{*}{ Variables $^{\mathrm{a}}$} & \multicolumn{3}{|l|}{ Use of health information technology } \\
\hline & $\begin{array}{l}\text { Dependent variable: fill prescription } \\
(\mathrm{n}=34,483), \mathrm{OR}^{\mathrm{b}}(95 \% \mathrm{CI})\end{array}$ & $\begin{array}{l}\text { Dependent variable: schedule medical } \\
\text { appointment ( } \mathrm{n}=34,477) \text {, OR ( } 95 \% \\
\text { CI) }\end{array}$ & $\begin{array}{l}\text { Dependent variable: email provider } \\
(\mathrm{n}=34,480) \text {, OR }(95 \% \mathrm{CI})\end{array}$ \\
\hline US-born non-Hispanic white & 1.00 (Reference) & 1.00 (Reference) & 1.00 (Reference) \\
\hline US-born Mexican & $1.01(0.81-1.26)$ & $1.01(0.83-1.23)$ & $0.85(0.68-1.06)$ \\
\hline US-born Puerto Rican & $0.94(0.53-1.68)$ & $1.12(0.71-1.77)$ & $0.8(0.5-1.27)$ \\
\hline $\begin{array}{l}\text { US-born Central and South Amer- } \\
\text { ican }\end{array}$ & $1.15(0.67-2)$ & $1.21(0.75-1.96)$ & $1.1(0.63-1.9)$ \\
\hline US-born other Latino & $0.48(0.2-1.15)^{\mathrm{c}}$ & $0.84(0.44-1.59)^{\mathrm{c}}$ & $0.43(0.2-0.93)^{\mathrm{c}}$ \\
\hline Foreign-born Mexican & $0.72(0.51-1.01)$ & $0.86(0.64-1.15)$ & $0.51(0.36-0.72)^{\mathrm{d}}$ \\
\hline Foreign-born Puerto Rican & $0.74(0.41-1.34)$ & $0.86(0.51-1.46)$ & $0.57(0.32-1)^{\mathrm{c}}$ \\
\hline $\begin{array}{l}\text { Foreign-born Central and South } \\
\text { American }\end{array}$ & $0.61(0.41-0.92)^{\mathrm{d}}$ & $0.82(0.59-1.13)$ & $0.7(0.5-0.99)^{\mathrm{d}}$ \\
\hline Foreign-born other Latino & $0.26(0.1-0.68)^{\mathrm{c}}$ & $0.6(0.32-1.12)^{\mathrm{c}}$ & $0.57(0.27-1.17)^{\mathrm{c}}$ \\
\hline
\end{tabular}

${ }^{\mathrm{a} C}$ Control variables not listed include age, sex, education, income to federal poverty level ratio, occupational group, insurance status, daily internet use, and region.

${ }^{\mathrm{b}} \mathrm{OR}$ : odds ratio.

${ }^{\mathrm{c}}$ Too few individuals use the technology in question to draw a statistical conclusion.

${ }^{\mathrm{d}}$ Indicates values are significant. 


\section{Posthoc Analysis}

We chose to analyze Mexican-identified individuals because they are the largest Hispanic ethnic subgroup in the United States [34] and contain enough individuals to do a subgroup analysis of a single Latino subethnic group $(\mathrm{N}=5310)$. As age is thought to be a factor in the use of social media and other Web-based communication channels [35], we conducted 2 posthoc analyses of Mexican-identified individuals to see if age was significant in predicting internet use, online HISB, or the use of HIT. We also conducted a third posthoc analysis to see if nativity and insurance status were significant predictors of online HISB and patient portal use among Mexican-identified individuals who used the internet. The supplementary file for posthoc analyses is provided in Multimedia Appendix 1.

\section{Examining Ages 18 to 30 Years}

We first examined Mexican-identified individuals to see if individuals aged 18 to 30 years behaved differently than individuals aged 31 years or older (posthoc analysis tables available from author on request). We found that those aged 18 to 30 year had higher odds of using the internet (OR 3.46, 95\% CI 2.61-4.59). Among individuals who used the internet, individuals aged 18 to 30 years had lower odds of looking up health information online (OR 0.75, 95\% CI 0.58-0.96), filling prescriptions online (OR $0.45,95 \%$ CI $0.27-0.75$ ), and using a chat group to learn about health topics (OR 0.55, 95\% CI $0.32-0.96$ ) than individuals aged 31 or older. There was no significant difference between these 2 age groups with regard to emailing a health care provider (OR 0.64, 95\% CI 0.4-1.02).

\section{Examining Ages 55 Plus}

We then examined Mexicans who used the internet to see if older individuals (aged 55 years or older) were significantly different than other age groups. Individuals who were 55 years or older had lower odds of using the internet (OR 0.13, 95\% CI $0.1-0.17)$ and higher odds of filling prescriptions online (OR $1.91,95 \%$ CI 1.17-3.14). There was no significant difference between individuals who were older than 55 years and individuals aged 18 to 54 years with respect to looking up health information online (OR 0.94, 95\% CI 0.71-1.23), scheduling a medical appointment online (OR 1.25, 95\% CI 0.77-2.01), emailing a health care provider (OR 1.66, 95\% CI 0.97-2.85), or using a chat group to learn about health topics (OR 2.02, 95\% CI 0.97-4.18).

\section{Within-Group Nativity Differences}

We then examined online HISB among Mexican-identified individuals who used the internet to understand differences in online HISB and patient portal use by nativity. We found that US-born individuals had higher odds (OR 52.9, 95\% CI 1.2-1.93) of looking up health information online compared with foreign-born individuals. There was no significant difference between US- and foreign-born Mexicans regarding the use of chat groups to learn about a health topic. US-born Mexican-identified individuals had significantly higher odds of using a computer to fill a prescription (OR 1.58, 95\% CI $1.02-2.44$ ) or emailing a provider (OR $1.73,95 \%$ CI 1.13-2.64) than foreign-born Mexican-identified individuals. There was no significant difference between US- and foreign-born
Mexicans who used the internet with regard to scheduling a medical appointment online. When we examined the relationship between having insurance and HISB or patient portal use among all Mexican-identified individuals who used the internet, we found that individuals who were insured had higher odds of filling prescriptions online (OR 2.66, 95\% CI 1.35-5.25), making medical appointments online (OR 2.08, 95\% CI 1.25-3.47), or using email to contact their provider (OR 2.45, 95\% CI 1.35-4.46).

\section{Discussion}

\section{Principal Findings}

Our findings show that although the use of Web-based patient portals is growing [1], there are significant disparities in online HISB and patient portal use in the United States between NH whites and various Latino subgroups after controlling for individual-level differences; however, these disparities vary by subgroup affiliation, nativity, and mode of access. Our findings also imply a gap in access between NH whites and the Mexican subpopulation as a whole, whereas foreign-born groups experience similar disparities across ethnic groups. Although recent reports have shown that internet access among foreign-born Latinos is increasing [17,33], our data show that the Mexican population and foreign-born Latinos in general should be a target for increased access.

Our findings regarding online HISB and patient portals are in line with previous literature showing low internet efficacy among Latinos as compared with whites $[8,9,13,36]$ and showing that Latinos may be less likely to use patient portals [4,37]. However, we expand upon previous research by showing that mode of access, nativity, and subgroup affiliation matter. Not all internet channels are equally likely to reach subpopulations within the Latino population. The need to pay attention to nativity and subgroup or regional affiliation among Latinos is particularly important in light of other studies that show that the internet is becoming the most common source of health information for all ethnic groups [38]. In addition, although emerging research shows that Spanish speakers view Web-based health portals positively [39] and computer-assisted interventions are being deployed more frequently among US Latinos [40], our research implies that the deployment of such interventions online will have uneven penetration among Latinos, particularly foreign-born individuals and Mexican-identified persons. Our findings also support previous research showing that certain portions of the US Mexican-identified population, such as farmworkers, do not seek health information online [36].

Generally, except for US-born Central and South Americans, our respondents are not looking up health information at the same rate as NH whites, although this result is only statistically significant for some groups. The pattern of the direction of the findings for using chat groups is the same for all groups except US-born Puerto Ricans, US-born Central and South Americans, and US- and foreign-born other Latinos. It is only significant for foreign-born Central and South Americans such that they are more likely than $\mathrm{NH}$ whites. It may be that this group uses chat groups that allow them to maintain relationships with social 
networks at home, perhaps allowing people to have contact with others from a similar cultural background. Our data suggest a trend where foreign-born Latinos use patient portals less often than US-born NH whites; however, the difference is only significant for some groups. There are a number of different possible reasons for this. Latinos who are less comfortable with English trust mediated sources less [41]. Previous literature has shown that Latinos may rely on and prefer interpersonal channels [42,43], and 1 study suggested that although some Latinos view patient portals positively, they are less interested in accessing their own medical records online [44], whereas an analysis of focus groups found that Latinos preferred portals that were easy to use and accessible on a mobile phone [43]. Our findings suggest that medical care providers could encourage the use of portals by their patients from these subpopulations by providing handouts, short conversations during appointments, or other aids to encourage use. Alternative forms of patient portals, including portals that are in Spanish, culturally tailored, encourage more personal forms of patient-provider interactions and are easily accessible on mobile phones, which may be a more effective way to reach this target population.

\section{Mexican-Identified Subpopulation}

Our posthoc analysis of the Mexican-identified subpopulation shows that nativity also matters within Latinos groups, which is in line with other studies that use Spanish as a measure of acculturation $[5,36]$ and with previous state-level studies showing differences between US- and foreign-born Latinos [9]. US-born individuals within the Mexican subethnicity were generally more likely to use HISB or patient portals than their foreign-born counterparts (although this was not true across the board), which is in line with other studies that show that more acculturated Mexican Americans are willing to use patient portals [44]. Among Mexican-identified persons, individuals who were insured had higher odds of using patient portals more within this group, which may indicate that access to health care is an important factor for patient portal use among Mexican Americans.

\section{Age Effects}

Previous studies have shown that individuals who are younger than 35 years are less likely to use patient portals [4], and our posthoc analysis found that younger Mexicans had lower odds of looking up health information online, filling prescriptions online, scheduling a medical appointment online, and using a chat group to learn about health topics, which may be because of the fact that younger individuals have less health problems than older individuals or that individuals with multiple chronic conditions are more likely to use patient portals than those without chronic conditions $[45,46]$. Although some studies have shown that older Latino adults were less likely to have activated patient portals as compared with $\mathrm{NH}$ whites [16], we found that among Mexican-identified individuals, people aged 55 years or older had higher odds of filling prescriptions online, which may be related to their health status. Future research should examine which Web-based apps, programs, and services are being used, especially by foreign-born Latinos and the Mexican-identified population. Such research will allow effective dissemination of health information to foreign-born individuals and potentially reach individuals who are not using other avenues to obtain health information.

\section{Limitations}

As this is a secondary data analysis of information, the primary limitation is the use of cross-sectional data, which means there is no causal analysis possible. The data are self-reported; therefore, recall bias and other factors may affect measurement. Furthermore, literacy significantly influences online behavior [47-49], and no measures of online literacy, including health or computer literacy, are assessed in the NHIS. For some groups, too few people reported engaging in HIT to report the findings as statistically significant; consequently, in 1 of our posthoc analyses, we combined US- and foreign-born individuals. Furthermore, as the questions on internet use, HISB, and HIT were asked to all respondents, it was possible that the respondents' answers were inconsistent, where they could answer that they did not use the internet but report that they emailed their provider.

\section{Conclusions}

Our findings indicate that the significant gaps in online health-related behaviors between $\mathrm{NH}$ whites and Latinos vary by subgroup affiliation, nativity, and mode of access. Although foreign-born Latinos of all ethnic subgroups have lower rates of online HISB, US-born Mexicans also experience lower odds than US-born NH whites. This is important in terms of information dissemination because it suggests that many of the channels that are increasingly being used by $\mathrm{NH}$ whites are not effective for communicating with many Latino groups. In addition, our results highlight the need for additional training in internet and patient portal technologies as health information becomes increasingly available online for many of the groups in this study. Without additional training and given that there is such a variation in health behaviors and outcomes, care should be taken when deciding which online channels will be used to reach these populations.

\section{Conflicts of Interest}

None declared.

\section{Multimedia Appendix 1}

Supplementary file for posthoc analyses.

[PDF File (Adobe PDF File), 123KB-Multimedia Appendix 1]

\section{References}


1. Cohen RA, Stussman B. Centers for Disease Control and Prevention. Health Information Technology Use Among Men and Women Aged 18-64: Early Release of Estimates From the National Health Interview Survey, January-June 2009 URL: https://www.cdc.gov/nchs/data/hestat/healthinfo2009/healthinfo2009.pdf[WebCite Cache ID 73MbrcCfp]

2. McCann E. Healthcare IT News. Most providers now using patient portals URL: http://www.healthcareitnews.com/news/ most-providers-now-using-patient-portals [accessed 2018-03-13] [WebCite Cache ID 6xsJ8I51s]

3. Kruse CS, Argueta DA, Lopez L, Nair A. Patient and provider attitudes toward the use of patient portals for the management of chronic disease: a systematic review. J Med Internet Res 2015;17(2):e40 [FREE Full text] [doi: 10.2196/jmir.3703] [Medline: 25707035]

4. Irizarry T, DeVito DA, Curran CR. Patient portals and patient engagement: a state of the science review. J Med Internet Res 2015;17(6):e148 [FREE Full text] [doi: 10.2196/jmir.4255] [Medline: 26104044]

5. De Jesus M, Xiao C. Predicting internet use as a source of health information: a "Language Divide" among the Hispanic population in the United States. Policy Internet 2012;4(2):1-11 [FREE Full text] [doi: 10.1515/1944-2866.1178]

6. Choi NG, Dinitto DM. The digital divide among low-income homebound older adults: internet use patterns, eHealth literacy, and attitudes toward computer/internet use. J Med Internet Res 2013 May;15(5):e93 [FREE Full text] [doi: 10.2196/jmir.2645] [Medline: 23639979]

7. Cunningham PJ, Hibbard J, Gibbons CB. Raising low 'patient activation' rates among Hispanic immigrants may equal expanded coverage in reducing access disparities. Health Aff (Millwood) 2011 Oct;30(10):1888-1894 [FREE Full text] [doi: 10.1377/hlthaff.2009.0805] [Medline: 21976331]

8. Brown A, Lopez G, Lopez M. Pew Research Center. 2016. Digital Divide Narrows for Latinos as More Spanish Speakers and Immigrants Go Online URL: http://tinyurl.com/htp3jnv [accessed 2018-08-31] [WebCite Cache ID 6xsIe36Js]

9. Gonzalez M, Sanders-Jackson A, Emory J. Online health information-seeking behavior and confidence in filling out online forms among Latinos: a cross-sectional analysis of the California Health Interview Survey, 2011-2012. J Med Internet Res 2016 Jul 04;18(7):e184 [FREE Full text] [doi: 10.2196/jmir.5065] [Medline: 27377466]

10. Chaet AV, Morshedi B, Wells KJ, Barnes LE, Valdez R. Spanish-language consumer health information technology interventions: a systematic review. J Med Internet Res 2016 Dec 10;18(8):e214 [FREE Full text] [doi: 10.2196/jmir.5794] [Medline: 27511437]

11. Christopher Gibbons M. Use of health information technology among racial and ethnic underserved communities. Perspect Health Inf Manag 2011;8:1f [FREE Full text] [Medline: 21307989]

12. Perrin A. Pew Research Center. Smartphones help blacks, Hispanics bridge some-but not all-digital gaps with whites URL: http://tinyurl.com/ya8bdrkz [accessed 2018-10-15] [WebCite Cache ID 73CLXer5n]

13. López L, Grant RW. Closing the gap: eliminating health care disparities among Latinos with diabetes using health information technology tools and patient navigators. J Diabetes Sci Technol 2012 Jan;6(1):169-176 [FREE Full text] [doi: 10.1177/193229681200600121] [Medline: 22401336]

14. Peña-Purcell N. Hispanics' use of internet health information: an exploratory study. J Med Libr Assoc 2008 Apr;96(2):101-107 [FREE Full text] [doi: 10.3163/1536-5050.96.2.101] [Medline: 18379664]

15. Goldzweig CL, Orshansky G, Paige NM, Towfigh AA, Haggstrom DA, Miake-Lye I, et al. Electronic patient portals: evidence on health outcomes, satisfaction, efficiency, and attitudes: a systematic review. Ann Intern Med 2013 Nov 19;159(10):677-687. [doi: 10.7326/0003-4819-159-10-201311190-00006] [Medline: 24247673]

16. Gordon NP, Hornbrook MC. Differences in access to and preferences for using patient portals and other ehealth technologies based on race, ethnicity, and age: a database and survey study of seniors in a large health plan. J Med Internet Res 2016 Mar 04;18(3):e50 [FREE Full text] [doi: 10.2196/jmir.5105] [Medline: 26944212]

17. Sarkar U, Karter AJ, Liu JY, Adler NE, Nguyen R, López A, et al. Social disparities in internet patient portal use in diabetes: evidence that the digital divide extends beyond access. J Am Med Inform Assoc 2011 May 1;18(3):318-321 [FREE Full text] [doi: 10.1136/jamia.2010.006015] [Medline: 21262921]

18. Wallace LS, Angier H, Huguet N, Gaudino JA, Krist A, Dearing M, et al. Patterns of electronic portal use among vulnerable patients in a nationwide practice-based research network: From the OCHIN Practice-Based Research Network (PBRN). J Am Board Fam Med 2016 Oct;29(5):592-603 [FREE Full text] [doi: 10.3122/jabfm.2016.05.160046] [Medline: 27613792]

19. Finney Rutten LJ, Hesse BW, Moser RP, Ortiz Martinez AP, Kornfeld J, Vanderpool RC, et al. Socioeconomic and geographic disparities in health information seeking and internet use in Puerto Rico. J Med Internet Res 2012;14(4):e104 [FREE Full text] [doi: 10.2196/jmir.2007] [Medline: 22849971]

20. Lee YJ, Boden-Albala B, Larson E, Wilcox A, Bakken S. Online health information seeking behaviors of Hispanics in New York City: a community-based cross-sectional study. J Med Internet Res 2014;16(7):e176 [FREE Full text] [doi: 10.2196/jmir.3499] [Medline: 25092120]

21. Lee YJ, Boden-Albala B, Quarles L, Wilcox A, Bakken S. Predictors of health information-seeking behaviors in Hispanics. NI 2012 (2012) 2012;2012:243 [FREE Full text] [Medline: 24199094]

22. Selsky C, Luta G, Noone A, Huerta EE, Mandelblatt JS. Internet access and online cancer information seeking among Latino immigrants from safety net clinics. J Health Commun 2013;18(1):58-70 [FREE Full text] [doi:

10.1080/10810730.2012.688248] [Medline: 23066874] 
23. Stepler R, Lopez M. Pew Research Center. US Latino Population Growth and Dispersion Has Slowed Since Onset of the Great Recession URL: http://tinyurl.com/jz8yh66 [accessed 2018-10-21] [WebCite Cache ID 6xsHS7h1o]

24. Patten E. Pew Research Center. The Nation's Latino Population is Defined by its Youth Nearly Half of US-Born Latinos Are Younger Than 18 URL: http://www.pewresearch.org/wp-content/uploads/sites/5/2016/04/ PH 2016-04-20 LatinoYouth-Final.pdf [accessed 2018-10-16] [WebCite Cache ID 73DxLdtPw]

25. Aponte J. Diabetes-related risk factors across Hispanic subgroups in the Hispanic health and nutritional examination survey (1982-1984). Public Health Nurs 2009;26(1):23-38. [doi: 10.1111/j.1525-1446.2008.00741.x] [Medline: 19154190]

26. Oquendo MA, Lizardi D, Greenwald S, Weissman MM, Mann JJ. Rates of lifetime suicide attempt and rates of lifetime major depression in different ethnic groups in the United States. Acta Psychiatr Scand 2004 Dec;110(6):446-451. [doi: 10.1111/j.1600-0447.2004.00404.x] [Medline: 15521829]

27. Kerner JF, Breen N, Tefft MC, Silsby J. Tobacco use among multi-ethnic Latino populations. Ethn Dis 1998;8(2):167-183. [Medline: 9681283]

28. Fox RS, Mills SD, Roesch SC, Sotres-Alvarez D, Gonzalez P, Bekteshi V, et al. Perceptions of cancer risk/efficacy and cancer-related risk behaviors: results from the HCHS/SOL Sociocultural Ancillary Study. Health Educ Behav 2018 Oct;45(5):790-799. [doi: 10.1177/1090198117744242] [Medline: 29284295]

29. Fox S, Livingston G. Pew Research Center. 2018. Latinos Online URL: http://www.pewhispanic.org/2007/03/14/ latinos-online/ [accessed 2018-10-15] [WebCite Cache ID 73DxrtF6x]

30. Center for Disease Control and Prevention. Centers for Disease Control and Prevention. 2015 NHIS Questionnaire - Sample Adult URL: ftp://ftp.cdc.gov/pub/Health_Statistics/NCHS/Survey_Questionnaires/NHIS/2015/english/qadult.pdf[WebCite Cache ID 6xsJq2D72]

31. Pew Research Center. 2013. Second-Generation Americans: A Portrait of the Adult Children of Immigrants URL: http:/ /www.pewsocialtrends.org/2013/02/07/second-generation-americans/ [accessed 2018-03-04] [WebCite Cache ID 6xsK7zKpP]

32. Pew Research Center. 2013. Between Two Worlds: How Young Latinos Come of Age in America URL: http://tinyurl.com/ yyrpj4j2 [accessed 2018-03-03] [WebCite Cache ID 6xsKWp8s3]

33. Brown A, Lopez G, Lopez M. Pew Research Center. 2016. Internet use among Hispanics URL: http://www.pewhispanic.org/ 2016/07/20/1-internet-use-among-hispanics/ [accessed 2018-03-05] [WebCite Cache ID 6xsGyUHsk]

34. Flores A. Pew Research Center. 2017. How the US Hispanic population is changing URL: http://www.pewresearch.org/ fact-tank/2017/09/18/how-the-u-s-hispanic-population-is-changing/ [accessed 2018-10-29] [WebCite Cache ID 73XXuuHfK]

35. Pew Research Center. 2017. Social media use by age URL: http://www.pewinternet.org/chart/social-media-use-by-age/ [accessed 2018-10-29] [WebCite Cache ID 73XY4gcsM]

36. Ginossar T. Predictors of online cancer prevention information seeking among patients and caregivers across the digital divide: a cross-sectional, correlational study. JMIR Cancer 2016 Mar 09;2(1):e2 [FREE Full text] [doi: 10.2196/cancer.5108] [Medline: 28410177]

37. Ancker JS, Hafeez B, Kaushal R. Socioeconomic disparities in adoption of personal health records over time. Am J Manag Care 2016 Aug;22(8):539-540 [FREE Full text] [Medline: 27541700]

38. Nguyen AB, Robinson J, O'Brien EK, Zhao X. Racial and ethnic differences in tobacco information seeking and information sources: findings from the 2015 Health Information National Trends Survey. J Health Commun 2017 Sep;22(9):743-752. [doi: 10.1080/10810730.2017.1347216] [Medline: 28762887]

39. Ochoa A, Kitayama K, Uijtdehaage S, Vermillion M, Eaton M, Carpio F, et al. Patient and provider perspectives on the potential value and use of a bilingual online patient portal in a Spanish-speaking safety-net population. J Am Med Inform Assoc 2017 Nov 01;24(6):1160-1164. [doi: 10.1093/jamia/ocx040] [Medline: 28460130]

40. Klein CH, Kuhn T, Altamirano M, Lomonaco C. C-SAFE: a computer-delivered sexual health promotion program for Latinas. Health Promot Pract 2017 Dec;18(4):516-525. [doi: 10.1177/1524839917707791] [Medline: 28490203]

41. Clayman ML, Manganello JA, Viswanath K, Hesse BW, Arora NK. Providing health messages to Hispanics/Latinos: understanding the importance of language, trust in health information sources, and media use. J Health Commun 2010;15(Suppl 3):252-263 [FREE Full text] [doi: 10.1080/10810730.2010.522697] [Medline: 21154097]

42. Elder JP, Ayala GX, Parra-Medina D, Talavera GA. Health communication in the Latino community: issues and approaches. Annu Rev Public Health 2009;30:227-251. [doi: 10.1146/annurev.publhealth.031308.100300] [Medline: 19296776]

43. Lyles CR, Allen JY, Poole D, Tieu L, Kanter MH, Garrido T. "I Want to Keep the Personal Relationship With My Doctor": understanding barriers to portal use among African Americans and Latinos. J Med Internet Res 2016 Oct 03;18(10):e263 [FREE Full text] [doi: 10.2196/jmir.5910] [Medline: 27697748]

44. Trubitt M, Alozie O, Shokar G, Flores S, Shokar NK. Patterns and correlates of internet use, cell phone use, and attitudes toward patient portals among a predominantly Mexican-American clinic population. Telemed J E Health 2018;24(11):861-869. [doi: 10.1089/tmj.2017.0266] [Medline: 29393769]

45. Greenberg AJ, Falisi AL, Finney Rutten LJ, Chou WS, Patel V, Moser RP, et al. Access to electronic personal health records among patients with multiple chronic conditions: a secondary data analysis. J Med Internet Res 2017 Jun 02;19(6):e188 [FREE Full text] [doi: 10.2196/jmir.7417] [Medline: 28576755]

46. Hopman WM, Harrison MB, Coo H, Friedberg E, Buchanan M, VanDenKerkhof EG. Associations between chronic disease, age and physical and mental health status. Chronic Dis Can 2009;29(3):108-116 [FREE Full text] [Medline: 19527569] 
47. Cline RJ, Haynes KM. Consumer health information seeking on the internet: the state of the art. Health Educ Res 2001 Dec;16(6):671-692 [FREE Full text] [Medline: 11780707]

48. Dincer S. A study of the relationship between pupils and parents' computer literacy level and use. Procedia Social Behav Sci 2012;46:484-489 [FREE Full text] [doi: 10.1016/j.sbspro.2012.05.146]

49. Coughlin SS, Stewart JL, Young L, Heboyan V, De Leo G. Health literacy and patient web portals. Int J Med Inform 2018 May;113:43-48. [doi: 10.1016/j.ijmedinf.2018.02.009] [Medline: 29602432]

\title{
Abbreviations
}

FPL: federal poverty level

HISB: health information-seeking behavior

HIT: health information technology

NH: non-Hispanic

NHIS: National Health Interview Survey

OR: odds ratio

\author{
Edited by G Eysenbach; submitted 13.03.18; peer-reviewed by A Nguyen, $K$ Fuji; comments to author 30.07.18; revised version \\ received 31.10.18; accepted 12.12.18; published 16.04.19 \\ Please cite as: \\ Gonzalez M, Sanders-Jackson A, Wright T \\ Web-Based Health Information Technology: Access Among Latinos Varies by Subgroup Affiliation \\ J Med Internet Res 2019;21(4):e10389 \\ URL: https://www.jmir.org/2019/4/e10389/ \\ doi: $\underline{10.2196 / 10389}$ \\ PMID: 30990462
}

CMariaelena Gonzalez, Ashley Sanders-Jackson, Tashelle Wright. Originally published in the Journal of Medical Internet Research (http://www.jmir.org), 16.04.2019. This is an open-access article distributed under the terms of the Creative Commons Attribution License (https://creativecommons.org/licenses/by/4.0/), which permits unrestricted use, distribution, and reproduction in any medium, provided the original work, first published in the Journal of Medical Internet Research, is properly cited. The complete bibliographic information, a link to the original publication on http://www.jmir.org/, as well as this copyright and license information must be included. 